\title{
Analytical ground state for the Jaynes-Cummings model with the ultrastrong coupling
}

\author{
Yuanwei Zhang, ${ }^{1,2}$ Gang Chen ${ }^{*},{ }^{1,3}$ Lixian Yu, ${ }^{3}$ Qifeng Liang, ${ }^{3}$ J. -Q. Liang, ${ }^{2}$ and Suotang Jia ${ }^{1}$ \\ ${ }^{1}$ State Key Laboratory of Quantum Optics and Quantum Optics Devices, \\ College of Physics and Electronic Engineering, Shanxi University, Taiyuan 030006, P. R. China \\ ${ }^{2}$ Institute of Theoretical Physics, Shanxi University, Taiyuan 030006, P. R. China \\ ${ }^{3}$ Department of Physics, Shaoxing University, Shaoxing 312000, P. R. China
}

\begin{abstract}
We present a generalized variational method to analytically obtain the ground-state properties of the unsolvable Jaynes-Cummings model with the ultrastrong coupling. An explicit expression for the ground-state energy, which agrees well with the numerical simulation in a wide range of the experimental parameters, is given. In particular, the introduced method can successfully solve this Jaynes-Cummings model with the positive detuning (the atomic resonant level is larger than the photon frequency), which can not be treated in the adiabatical approximation and the generalized rotating-wave approximation. Finally, we also demonstrate analytically how to control the mean photon number by means of the current experimental parameters including the photon frequency, the coupling strength, and especially the atomic resonant level.
\end{abstract}

PACS numbers: $42.50 . \mathrm{Pq}$

The Jaynes-Cummings model, which describes the important interaction between the atom and the photon of a quantized electromagnetic field, is a fundamental model in quantum optics and condensed-matter physics as well as in quantum information science. In the optical cavity quantum electrodynamics, the atom-photon coupling strength is far smaller than the photon frequency. As a result, the system dynamics can be well governed by the Jaynes-Cummings model with the rotating-wave approximation (RWA) [1]. In the case of the RWA, its energy spectrum and wavefunctions can be solved exactly [2]. With the rapid development of fabricated technique in solid-state systems, the Jaynes-Cummings model can be realized in semiconducting dots [3, 6] and superconducting Josephson junctions [7-12]. More importantly, recent experiment has reported the existence of the ultrastrong coupling with the ratio 0.12 between the coupling strength and the microwave photon frequency [13]. Moreover, this ratio maybe approach unit due to the current efforts [14, 15].

However, in this ultrastrong coupling regime the wellknown RWA breaks down and the whole Hamiltonian is written as

$$
H_{x}=\omega\left(a^{\dagger} a+\frac{1}{2}\right)+\frac{1}{2} \Omega \sigma_{z}+g\left(\sigma_{+}+\sigma_{-}\right)\left(a^{\dagger}+a\right),
$$

where $a^{\dagger}$ and $a$ are creation and annihilation operators for photon with frequency $\omega, \sigma_{ \pm}$are the raising and lowering operators of the two-level atom in the basis of $\sigma_{z}$, $\Omega$ is the atomic resonant frequency and $g$ is the atomphoton coupling strength. Due to the existence of the counter-rotating terms $\left(\sigma_{+} a^{+}\right.$and $\left.\sigma_{-} a\right)$, Hamiltonian (1) is very difficult to be solved analytically except for $\Omega=0$. Although the energy spectrum of Hamiltonian (1) has been obtained perfectly by means of the numerical simu-

${ }^{*}$ Corresponding author: chengang971@163.com lation 16 18], the analytical solutions are very necessary for extracting the fundamental physics as well as in processing quantum information [19 21]. In the negative detuning $\Omega<\omega$, the adiabatic approximation method that the second term of Hamiltonian (1) is treated as a small perturbation has been considered 22]. Recently, a generalized rotating-wave approximation (GRWA) has also been proposed to solve Hamiltonian (1) in the displaced oscillator basis states [23]. This method can derive an analytical energy of Hamiltonian (1) in the ultrastrong coupling successfully. However, it, like the adiabatic approximation method, is invalid in the case of the positive detuning $(\Omega>\omega)[24]$. Moreover, the GRWA also leads to an unphysical relation that the mean photon number is independent of the atomic resonant frequency. Thus, it is very necessary to put forward a new method to reconsider Hamiltonian (1).

In this brief paper we present a generalized variational method (GVM) to analytically obtain the ground-state properties of Hamiltonian (1) in the ultrastrong coupling regime [13]. An explicit expression for the ground-state energy, which agrees well with the numerical simulation in a wide range of the experimental parameters, is given. More importantly, our method is valid for all regions of the atomic resonant level including the negative detuning $(\Omega<\omega)$, the resonant case $(\Omega=\omega)$, and especially the positive detuning $(\Omega>\omega)$. Finally, we also demonstrate analytically that the mean photon number is strongly dependent on all parameters of Hamiltonian (1) including the photon frequency, the coupling strength, and the atomic resonant level. For a weak atomic resonant frequency, the mean photon number depends linearly on it.

We first perform a rotation around the y-axis to rewrite Hamiltonian (1) as $H_{z}=\omega\left(a^{\dagger} a+\frac{1}{2}\right)+\frac{1}{2} \Omega \sigma_{x}-g \sigma_{z}\left(a^{\dagger}+a\right)$. Under a unitary transformation $U=\exp \left[\lambda \sigma_{z}\left(a^{\dagger}-a\right)\right]$, the above Hamiltonian $H_{z}$ becomes

$$
H_{u}=\lambda^{2} \omega+2 \lambda g+H_{a}+H_{g}+H_{\Omega}
$$

with $H_{a}=\omega\left(a^{\dagger} a+\frac{1}{2}\right), H_{g}=-(g+\lambda \omega)\left(a^{\dagger}+a\right) \sigma_{z}$, and 
$H_{\Omega}=\frac{1}{2} \Omega\left\{\sigma_{+} \exp \left[2 \lambda\left(a^{\dagger}-a\right)\right]+\sigma_{-} \exp \left[-2 \lambda\left(a^{\dagger}-a\right)\right]\right\}$, where $\lambda$ is a dimensionless parameter. In the JaynesCummings model with the RWA, the dimensionless parameter $\lambda$ can be determined by setting $H_{g}=0$ and an effective Hamiltonian can be obtained by series expansion with respect to $g / \omega$ [7]. However, in the ultrastrong coupling, this derivation is not valid. Moreover, the terms $H_{g}$ and $H_{\Omega}$ play the important role in the energy spectrum. As will be shown, the dimensionless parameter $\lambda$ in the ultrastrong coupling can be determined analytically.

We now choose the basis states $| \pm, N\rangle=$ $\frac{1}{\sqrt{2}}\left(c_{e}^{\dagger} \pm c_{g}^{\dagger}\right)|0\rangle_{a}|N\rangle$, where $c_{e}^{\dagger}$ and $c_{g}^{\dagger}$ are the creation operators of the excited- and ground- states, $|0\rangle_{a}$ is the vacuum state of atom and $|N\rangle$ is the Fock state of the photon, to calculate the energy spectrum by means of the perturbation method [25]. This method requires the diagonal and non-diagonal matrix elements of Hamiltonian (2) in the basis states. Since $-(g+\lambda \omega)\left\langle N, \pm\left|\left(a^{\dagger}+a\right) \sigma_{z}\right| N, \pm\right\rangle=0$ and $\omega\left\langle N, \pm\left|\left(a^{\dagger} a+\frac{1}{2}\right)\right| N, \pm\right\rangle=\omega\left(N+\frac{1}{2}\right)$, the diagonal part is given by

$$
H_{0}=\lambda^{2} \omega+2 \lambda g+\omega\left(N+\frac{1}{2}\right)+\left\langle H_{\Omega}\right\rangle
$$

with $\left\langle H_{\Omega}\right\rangle=\left\langle N, \pm\left|H_{\Omega}\right| N, \pm\right\rangle=\mp F(\lambda) L_{N}^{0}\left(4 \lambda^{2}\right)$, where $F(\lambda)=-\frac{1}{2} \Omega \exp \left(-2 \lambda^{2}\right)$ and $L_{i}^{j}(x)$ is the associated Laguerre polynomials. The non-diagonal parts must be divided into two cases including $\left\langle N, \pm\left|H_{g}\right| \pm, M\right\rangle$ and $\left\langle N, \pm\left|H_{\Omega}\right| \pm, M\right\rangle$ for even $(N-$ $M)$, and $\left\langle N, \pm\left|H_{g}\right| \mp, M\right\rangle$ and $\left\langle N, \pm\left|H_{\Omega}\right| \mp, M\right\rangle$ for odd $(N-M)$ since $\left\langle N, \pm\left|H_{a}\right| \pm, M\right\rangle=\left\langle N, \pm\left|H_{a}\right| \mp, M\right\rangle=0$. When $(N-M)$ is even, we have $\left\langle N, \pm\left|H_{g}\right| \pm, M\right\rangle=$ 0 and $\left\langle N, \pm\left|H_{\Omega}\right| \pm, M\right\rangle=P_{N, M}$, where $P_{N, M}=$ $\mp F(\lambda)(2 \lambda)^{N-M} \sqrt{M ! / N !} L_{M}^{N-M}\left(4 \lambda^{2}\right)$. When $(N-M)$ is odd, the matrix elements become $\left\langle N, \pm\left|H_{g}\right| \mp, M\right\rangle=$ $-\sqrt{N} \omega(g / \omega+\lambda) \delta_{N, M+1}$ and $\left\langle N, \pm\left|H_{\Omega}\right| \mp, M\right\rangle=$ $-P_{N, M}$. From the above calculation we assume $N>M$ in terms of the Hermitian of Hamiltonian (2).

By means of the matrix form of Hamiltonian (2) with the derived diagonal and non-diagonal terms, the energy spectrum and wavefunction can, in principle, be obtained. However, the procedure is very complicated. Fortunately, in the ultrastrong coupling regime, the groundstate properties can be calculated by the perturbation expansion of the non-diagonal terms. In this method, the diagonal part is regarded as the unperturbed Hamiltonian $H_{0}$ and the remaining part of Hamiltonian, $H_{r}=$ $H_{u}-H_{0}$ is treated as a perturbation [25]. [26]

Formally, the ground-state energy can be written as

$$
E_{0}=E_{0}^{(0)}+E_{0}^{(1)}+E_{0}^{(2)}
$$

Here $E_{0}^{(0)}$ is the unperturbed ground-state energy. According to Eq. (3), this unperturbed ground-state energy is given by $E_{0}^{(0)}=\frac{1}{2} \omega+\lambda^{2} \omega+2 \lambda g+F(\lambda)$ for $N=0$.

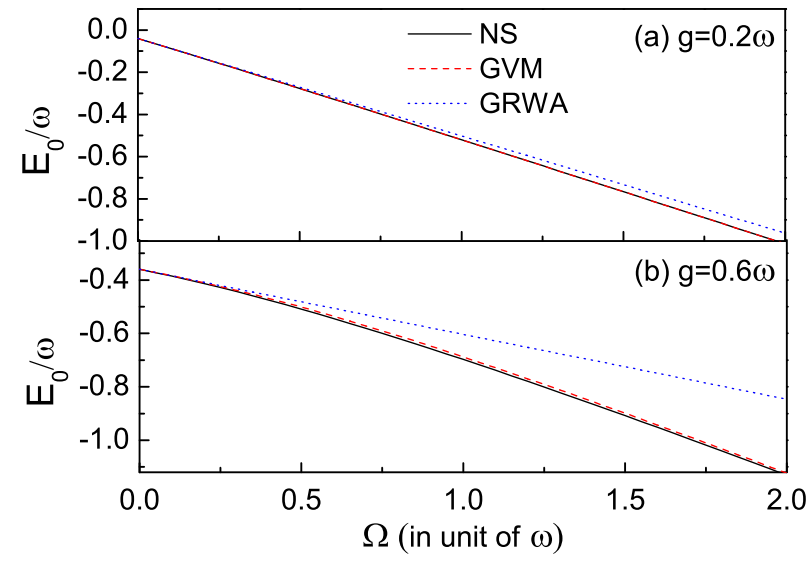

FIG. 1: (Color online) The ground-state energy $E_{0}$ as a function of the atomic resonant frequency $\Omega$ for the different coupling strength $g=0.2 \omega$ (a) and $0.6 \omega$ (b) by means of the numerical simulation (NS), the GVM and the GRWA.

The first-order correction to the unperturbed groundstate energy is given by $E_{0}^{(1)}=0$ since $H_{r}$ has no diagonal matrix element. While the second-order correction is evaluated as $E_{0}^{(2)}=-[-(g+\omega \lambda)+2 \lambda F(\lambda)]^{2} /[\omega-$ $\left.2 F(\lambda)\left(1-2 \lambda^{2}\right)\right]-\sum_{N=2}^{\infty} F^{2}(\lambda)(2 \lambda)^{2 N} / N !\left[E_{e, N}^{(0)}-E_{0}^{(0)}\right]$, where $e= \pm N$ is odd (even). On the other hand, according to the spirit of the variational method [25], the dimensionless parameter $\lambda$ can be derived from minimizing the unperturbed ground-state energy $E_{0}^{(0)}$ by

$$
\lambda\left[\omega+\Omega \exp \left(-2 \lambda^{2}\right)\right]+g=0 .
$$

By using the same procedure, the ground-state wavefunction is written by [26]

$$
\left|\Psi_{0}\right\rangle=\left|\Psi_{0}^{(0)}\right\rangle+\left|\Psi_{0}^{(1)}\right\rangle
$$

where the unperturbed ground-state wavefunction is given by $\left|\Psi_{0}^{(0)}\right\rangle=|-, 0\rangle$, and the first-order correction to the unperturbed ground-state wavefunction is given by $\left|\Psi_{0}^{(1)}\right\rangle=-\frac{[-(g+\omega \lambda)+2 \lambda F(\lambda)]}{\omega-2 F(\lambda)\left(1-2 \lambda^{2}\right)}|+, 1\rangle-$ $\sum_{N=2,4, \cdots}^{\infty} \frac{F(\lambda)(2 \lambda)^{N}}{\sqrt{N !}\left[E_{-, N}^{(0)}-E_{0}^{(0)}\right]}|-, N\rangle$ $\sum_{N=3,5, \cdots}^{\infty} \frac{F(\lambda)(2 \lambda)^{N}}{\sqrt{N !}\left[E_{+, N}^{(0)}-E_{0}^{(0)}\right]}|+, N\rangle$.

Equations (4) and (6), together with Eq. (5), are the main results in this brief paper. Interestingly, the dimensionless parameter $\lambda$ depends on all parameters including the photon frequency $\omega$, the coupling strength $g$, and especially the atomic resonant frequency $\Omega$. In general, Eq. (5) can not be solved and the ground-state properties can not be obtained analytically due to the existence the complex sums in the expressions $E_{0}^{(2)}$ and $\left|\Psi_{0}^{(1)}\right\rangle$. However, in current experimental setups of the ultrastrong coupling (for example, $g \simeq 0.1 \omega$ in Ref. [13]), 


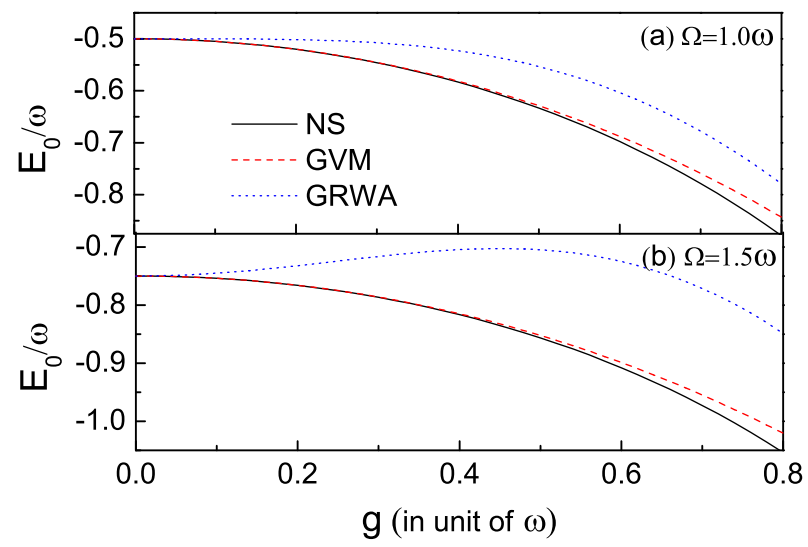

FIG. 2: (Color online) The ground-state energy $E_{0}$ as a function of the coupling strength $g$ for the different atomic resonant frequency $\Omega=1.0 \omega$ (a), and $1.5 \omega$ (b) by means of the numerical simulation (NS), the GVM, and the GRWA.

Eq. (5D) becomes very simple, and moreover, the complex sums are also simplified successfully. As a consequence, some interesting results, which govern the fundamental properties of Hamiltonian (1), can be achieved.

By means of Eq. (5), the dimensionless parameter $\lambda$ is derived approximately by $\lambda \simeq-g /\left\{\omega+\Omega \exp \left[-2 g^{2} /(\omega+\right.\right.$ $\left.\left.\Omega)^{2}\right]\right\}$. However, in current experimental setups of the ultrastrong coupling, $\exp \left[-2 g^{2} /(\omega+\Omega)^{2}\right] \simeq 1$, and thus, the dimensionless parameter $\lambda$ becomes

$$
\lambda=-\frac{g}{\omega+\Omega} .
$$

Based on Eq. (77), we have $E_{0}^{(0)}=\omega / 2-$ $g^{2}(\omega+2 \Omega) /(\omega+\Omega)^{2}-\Omega \exp \left[-2 g^{2} /(\omega+\Omega)^{2}\right] / 2$ and $E_{0}^{(2)} \quad \approx \quad-4 g^{2} \Omega^{2} \lambda^{4} /(\omega+\Omega)^{3} \quad-$ $\sum_{N=2}^{\infty} F^{2}(\lambda)(2 \lambda)^{2 N} / N !\left[E_{e, N}^{(0)}-E_{-, 0}^{(0)}\right]$. Due to the existence of the high order terms with respect to $\lambda$, the complex sum in $E_{0}^{(2)}$ can be omitted. Moreover, the first term of $E_{0}^{(2)}$ is far smaller than $E_{0}^{(0)}$. It means that the second-order perturbed energy almost has no effect to the ground-state energy. Therefore, an explicit ground-state energy for Hamiltonian (1) is given finally by

$$
E_{0} \simeq \frac{\omega}{2}-\frac{g^{2}(\omega+2 \Omega)}{(\omega+\Omega)^{2}}-\frac{\Omega}{2} \exp \left[-2\left(\frac{g}{\omega+\Omega}\right)^{2}\right] .
$$

For a weak atomic resonant frequency $(\Omega \ll \omega)$, Eq. (8) become $E_{0}^{G R W A}=1 / 2 \omega-g^{2} / \omega-1 / 2 \Omega \exp \left[-2(g / \omega)^{2}\right]$, which has been obtained perfectly by means of the GRWA [23].

Figure 1 shows the ground-state energy $E_{0}$ as a function of the atomic resonant frequency $\Omega$ for the different coupling strength $g=0.2 \omega$ and $0.6 \omega$ by means of the numerical simulation, the GVM $\left(E_{0}\right)$ and the GRWA $\left(E_{0}^{G R W A}\right)$. For a weak atomic resonant frequency $(\Omega<$

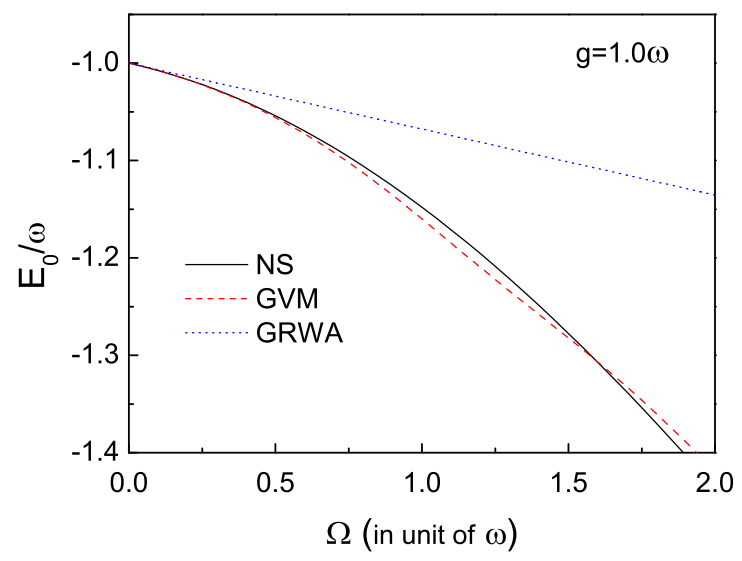

FIG. 3: (Color online) The ground-state energy $E_{0}$ as a function of the atomic resonant level $\Omega$ with the coupling strength $g=1.0 \omega$ by means of the numerical simulation (NS), the GVM, and the GRWA.

$\omega)$, both results derived from the GVM and the GRWA agree with the direct numerical simulation. In the positive detuning $(\Omega>\omega)$, the GRWA breaks down and the error is increased linearly. However, the result derived from the GVM can also agree well with that of the numerical simulation. Figure 2 shows the ground-state energy $E_{0}$ as a function of the coupling strength $g$ for different atomic resonant frequency $\Omega=1.0 \mathrm{\omega}$ and $1.5 \mathrm{\omega}$ by means of the numerical simulation, the GVM $\left(E_{0}\right)$ and the GRWA $\left(E_{0}^{G R W A}\right)$. This figure exhibits that the analytical expression in Eq. (8) can work reasonably for $g<0.8 \omega$. If $g \sim \omega$, Eq. (5) can not be solved explicitly and the complex sums in the expressions $E_{0}^{(2)}$ and $\left|\Psi_{0}^{(1)}\right\rangle$ can also not be simplified. However, the results in Eqs. (4) and (6) with the complex sums remain valid, as shown in Fig. 3.

Having obtained the ground-state energy, we now discuss the experimentally-measurable mean photon number $\left\langle a^{\dagger} a\right\rangle$, which can be obtained by $\left\langle a^{\dagger} a\right\rangle=$ $\left\langle\Psi_{0}\left|U a^{\dagger} a U^{\dagger}\right| \Psi_{0}\right\rangle=\left\langle\Psi_{0}\left|a^{\dagger} a+\lambda^{2}-\lambda \sigma_{z}\left(a^{\dagger}+a\right)\right| \Psi_{0}\right\rangle$. The expression of the mean photon number is very complex. However, here we give an approximate solution,

$$
\left\langle a^{\dagger} a\right\rangle \simeq \frac{g^{2}}{\left[\omega+\Omega \exp \left(-2 g^{2} / \omega^{2}\right)\right]^{2}} .
$$

Equation (9) demonstrates clearly that the mean photon number depends strongly on all parameters of Hamiltonian (11). For a weak atomic resonant frequency $(\Omega \ll \omega)$, the mean photon number becomes $\left\langle a^{\dagger} a\right\rangle=g^{2} / \omega^{2}-$ $2 g^{2} \Omega \exp \left(-2 g^{2} / \omega^{2}\right) / \omega^{3}$, which is quite different from the previous conclusion $\left\langle a^{\dagger} a\right\rangle_{G R W A}=g^{2} / \omega^{2}$ derived from the GRWA [23].

Figures 4 shows the mean photon number $\left\langle a^{\dagger} a\right\rangle$ as a function of the atomic resonant frequency $\Omega$ for the coupling strength $g=0.6 \omega$ by means of the numerical simulation, the GVM and the GRWA. In the insert of 


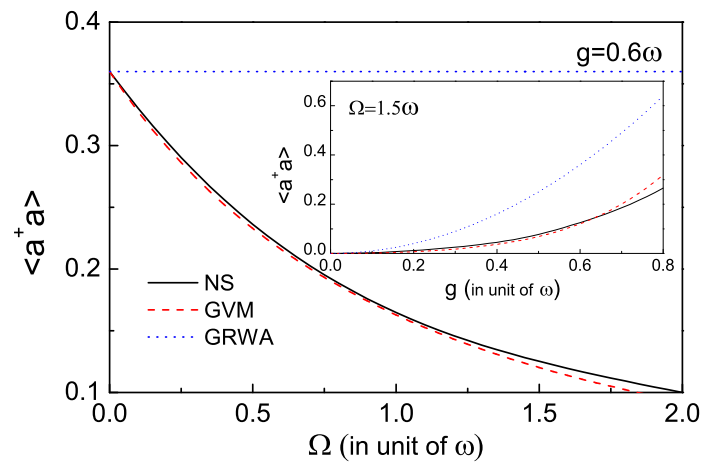

FIG. 4: (Color online) The mean photon number $\left\langle a^{\dagger} a\right\rangle$ as a function of the atomic resonant frequency $\Omega$ for the coupling strength $g=0.6 \omega$ by means of the numerical simulation (NS), the GVM, and the GRWA. Insert: The mean photon number $\left\langle a^{\dagger} a\right\rangle$ as a function of the coupling strength $g$ for the atomic resonant frequency $\Omega=1.5 \omega$.

Fig. 4, the mean photon number $\left\langle a^{\dagger} a\right\rangle$ as a function of the coupling strength $g$ is also plotted when the atomic resonant frequency is chosen as $\Omega=1.5 \omega$. Both these fig- ures show that our obtained explicit expression in Eq.(9) agrees well with the numerical simulation. It implies that the required mean photon number can controlled well by manipulating an external flux bias (the atomic resonant frequency), the inductive coupling of a qubit (the coupling strength), and a transmission line resonator (the photon frequency) in current experimental setups of the ultrastrong coupling [13].

In summary, we have presented the GVM to analytically obtain some interesting ground-state properties of the Jaynes-Cummings model with the ultrastrong coupling in all regions of the atomic resonant level including the negative detuning, the resonant case, and especially the positive detuning. However, for the excited state, this method is invalid since the second-order perturbation energy will be larger than the unperturbed groundstate energy.

We thank Drs. Chuanwei Zhang, Ming Gong, and Yongping Zhang for helpful discussions and suggestions. This work was supported partly by the NNSFC under Grant Nos. 10904092, 10934004, 60978018, 11074154, and 11075099, and the ZJNSF under Grant No. Y6090001.
[1] E. T. Jaynes, and F. W. Cummings, Proc. IEEE 51, 89 (1963).

[2] B. W. Shore, and P. L. Knight, J. Mod. Opt. 40, 1195 (1993).

[3] A. Imamoglu, D. D. Awschalom, G. Burkard, D. P. DiVincenzo, D. Loss, M. Sherwin, and A. Small, Phys. Rev. Lett. 83, 4204 (1999).

[4] G. Günter, A. A. Anappara, J. Hees, A. Sell1, G. Biasiol, L. Sorba, S. De Liberato, C. Ciuti, A. Tredicucci, A. Leitenstorfer, and R. Huber, Nature (London) 458, 178 (2009).

[5] A. A. Anappara, S. D. Liberato, A. Tredicucci, C. Ciuti, G. Biasio, L. Sorba, and F. Beltram, Phys. Rev. B 79, 201303, (2009)

[6] Y. Todorov, A. M. Andrews, R. Colombelli, S. De Liberato, C. Ciuti, P. Klang, G. Strasser, and C. Sirtori, Phys. Rev. Lett. 105, 196402 (2010).

[7] A. Blais, R.-S. Huang, A. Wallraff, S. M. Girvin, and R. J. Schoelkopf, Phys. Rev. A 69, 062320 (2004).

[8] A. Wallraff, D. I. Schuster, A. Blais, L. Frunzio, R.- S. Huang, J. Majer, S. Kumar, S. M. Girvin, and R. J. Schoelkopf, Nature (London) 431, 162 (2004).

[9] L. Tian, P. Rabl, R. Blatt, and P. Zoller, Phys. Rev. Lett. 92, 247902 (2004).

[10] M. Hofheinz, H. Wang, M. Ansmann, R. C. Bialczak, E. Lucero, M. Neeley, A. D. O'Connell, D. Sank, J. Wenner, John M. Martinis, and A. N. Cleland, Nature (London) 459, 546 (2009).

[11] M. D. LaHaye, J. Suh, P. M. Echternach, K. C. Schwab, and M. L. Roukes, Nature (London) 459, 960(2009).

[12] A. Fedorov, A. K. Feofanov, P. Macha, P. Forn-Díaz, C. J. P. M. Harmans, and J. E. Mooij, Phys. Rev. Lett. 105, 060503 (2010).
[13] T. Niemczyk, F. Deppe, H. Huebl, E. P. Menzel, F. Hocke, M. J. Schwarz, J. J. Garcia-Ripoll, D. Zueco, T. Hümmer, E. Solano, A. Marx, and R. Gross, Nature Physics 6, 772 (2010).

[14] J. Bourassa, J. M. Gambetta, A. A. Abdumalikov, Jr., O. Astafiev, Y. Nakamura, and A. Blais, Phys. Rev. A. 80, 032109 (2009).

[15] B. Peropadre, P. Forn-Díaz, E. Solano, and J. J. GarcíaRipoll, Phys. Rev. Lett. 105, 023601 (2010).

[16] I. D. Feranchuk, L. I. Komarov, and A. P. Ulyanenkov, J. Phys. A: Math. Gen. 29, 4035 (1996).

[17] Q. H. Chen, L. Li, T. Liu, and K. -L. Wang, arXiv: 1007.1747.

[18] Q. H. Chen, T. Liu, Y. -Y. Zhang, and K. -L. Wang, arXiv: 1011.3280 .

[19] H. Zheng, S. Y. Zhu, and M. S. Zubairy, Phys. Rev. Lett. 101, 200404 (2008).

[20] S. Ashhab, and F. Nori, Phys. Rev. A 81, 042311 (2010).

[21] Q. H. Chen,Y. Yang, T. Liu, and K. -L. Wang, Phys. Rev. A 82, 052306 (2010).

[22] E. K. Irish, J. Gea-Banacloche, I. Martin, and K. C. Schwa, Phys. Rev. B. 72, 195410 (2005).

[23] E. K. Irish, Phys. Rev. Lett. 99, 173601 (2007).

[24] J. Hausinger, and M. Grifoni, Phys. Rev. A 82, 062320 (2010).

[25] I. G. Lang, and Yu. A. Firsov, Zh. Eksp. Teor. Fiz. 43,1843 (1962) [Sov. Phys. JETP 16, 1301 (1963)].

[26] In this brief paper the ground-state energy is only considered up to the second order and the ground-state wavefunction is only considered up to the first order. These results can agree well with those of the numerical simulations. The detailed comparison is shown in Figs. (1)-(4). 